\title{
On the Condition Number of Local Bases for Piecewise Cubic Polynomials
}

\author{
By J. M. Varah*
}

\begin{abstract}
The condition number of the Gram matrix associated with piecewise polynomial finite element bases is discussed in general, and computed explicitly for cubic splines and cubic Hermite polynomials. In the latter case, we discuss the inherent ambiguity in the basis, and find the minimum condition number.
\end{abstract}

1. Introduction. There has been a great deal of interest lately in the approximate solution of various problems by so-called global methods: that is, to find a solution of the form

$$
\sum_{1}^{n} c_{i} \phi_{i}(x)
$$

where the $\phi_{i}(x)$ are given functions. Data fitting problems and boundary value problems have both been successfully treated in this way, particularly when the basis functions $\left\{\phi_{i}(x)\right\}$ are piecewise polynomials with support over a small region in $x$. We refer to Schultz [2] and Strang and Fix [3] for a general discussion of such methods.

Of crucial importance in computing with these bases is their condition, or "amount of linear dependence". This can be measured by the condition number of the Gram matrix (mass matrix in [3]):

$$
G_{i j}=\int \phi_{i}(x) \phi_{j}(x) d x
$$

Since $G$ is a positive definite symmetric matrix, the condition number in the $l_{2}$ norm is $k(G)=\lambda \max (G) / \lambda \min (G)$. We shall use this throughout the paper. Of course, if the basis functions are orthogonal over the whole $x$-domain, $k=1$; however, when we demand that the support of each basis function be restricted to a small region, we no longer have orthogonality, and the question of the condition of various bases becomes interesting.

In this paper we consider the condition of the most common piecewise polynomial bases: smooth cubic splines and piecewise cubic Hermite polynomials. For the former, this is merely a matter of direct computation; however, for the latter there is still some choice to be made, and we investigate the problem of minimizing the condition.

Note. As the referee points out, this condition, or amount of linear dependence, is more accurately measured by the square root of the condition number of the

Received May 6, 1975; revised June 7, 1976

AMS (MOS) subject classifications (1970). Primary 65L10, 65F35.

*Supported by NRC of Canada grant 67-8240. 
Gram matrix; although this does not change the results described in this paper greatly, it should be kept in mind.

2. The General Method. For a given general set of abscissas in the $x$-domain, the piecewise polynomial basis functions over these abscissas will vary throughout the interval and the Gram matrix, although banded, will have elements varying in size depending on the spacing of the abscissas. If we consider only equally spaced abscissas, then, as in [3, p. 209ff], the Gram matrix is Toeplitz, or block Toeplitz, and its condition is more readily discernable. Indeed, since the mesh spacing $h$ only appears as a common factor, it does not influence the condition, so the important consideration is the condition of the doubly infinite Toeplitz (or block-Toeplitz) Gram matrix. This corresponds to either a doubly infinite $x$-domain with fixed $h$, or a finite $x$-domain with $h \longrightarrow 0$.

Now let us concentrate on a $B$-spline basis; these are piecewise polynomials of degree $2 n-1$, and continuity $2 n-2$ at the abscissas, with support over $2 n$ intervals (see for example de Boor [1]). Since there is one basis function for each abscissa, each basis function $\phi_{i}(x)$ is a translated scaled copy of one basic function $S_{n}(x)$, centered at 0 with support $(-n, n)$. Thus, the Gram matrix $G_{n}$ has the form

$$
H_{n}=\frac{G_{n}}{h}=\left(\begin{array}{c}
a_{0} a_{1} \cdots a_{2 n-1} \\
a_{1} a_{0} \\
\vdots \\
a_{2 n-1} \\
0
\end{array}\right)
$$

where $a_{i}=\int S_{n}(x) S_{n}(x-i) d x, i=0, \ldots, 2 n-1$. The spectrum of the doubly infinite version of this Toeplitz matrix is well known:

$$
\operatorname{Sp}\left(H_{n}\right)=\left\{p(\theta)=a_{0}+2 \sum_{1}^{2 n-1} a_{j} \cos _{j} \theta\right\} .
$$

Thus $\kappa\left(H_{n}\right)=\max _{\theta} p(\theta) / \min _{\theta} p(\theta)$ and $0<\kappa\left(H_{n}\right)<\infty$ since $H_{n}$ is positive definite, so $p(\theta)>0$.

For $n=1$ (piecewise linear functions), $S_{n}(x)$ is the familiar roof function; as in [3, p. 211], we have $a_{0}=4, a_{1}=1$, and

$$
\kappa\left(H_{1}\right)=\frac{\max _{\theta}(4+2 \cos \theta)}{\min _{\theta}(4+2 \cos \theta)}=3 .
$$

For $n=2$ (cubic splines), $S_{2}(x)$ is the basic cubic $B$-spline (see Schultz [2, p. 73])

$$
S_{2}(x)= \begin{cases}(2-x)^{3}, & 1 \leqslant x \leqslant 2 \\ 3 x^{3}-6 x^{2}+4, & 0 \leqslant x \leqslant 1 \\ S(-x), & x<0\end{cases}
$$

A short computation gives 


$$
a_{0}=17 \frac{9}{35}, a_{1}=8 \frac{71}{140}, a_{2}=\frac{6}{7}, a_{3}=\frac{1}{140}
$$

The corresponding $p(\theta)$ has its maximum at $\theta=0: p(0)=36$, and its minimum at $\theta=\pi: p(\pi)=34 / 35$. Thus,

$$
\kappa\left(H_{2}\right)=\frac{315}{17} \cong 18.53
$$

In fact, these condition numbers for $B$-spline bases of smooth splines of any order can be easily derived from Schoenberg [4]:

$$
\kappa\left(H_{n}\right)=\frac{1 / 2(\pi / 2)^{4 n}}{\sum_{j=0}^{\infty}(2 j+1)^{-4 n}}=\frac{(4 n) !}{2^{4 n}\left(2^{4 n}-1\right) B_{n}},
$$

where $B_{n}$ is the $n$th Bernoulli number. Also, max norm condition numbers for the same bases are given in de Boor [1].

3. The Cubic Hermite Polynomials. The general piecewise Hermite polynomials have degree $2 n-1$ and continuity $n-1$ at the abscissas; because of this lower continuity, there are more basis functions: $n$ associated with each abscissa. The socalled natural Hermite basis, obtained by Hermite interpolation of the delta function at successive abscissas, gives the smallest support possible, namely two subintervals. For $n=2$, for equally spaced abscissas, the basis functions are translated scaled copies of two functions defined over $[-1,1]$ (see Schultz $[2$, p. 27]):

$$
H^{(0)}(x)= \begin{cases}(2 x+1)(x-1)^{2}, & 0 \leqslant x \leqslant 1, \\ H^{(0)}(-x), & -1 \leqslant x \leqslant 0,\end{cases}
$$

$$
H^{(1)}(x)= \begin{cases}x(x-1)^{2}, & 0 \leqslant x \leqslant 1 \\ -H^{(1)}(-x), & -1 \leqslant x \leqslant 0\end{cases}
$$

Of course, this is not the only basis with minimal support; we could use translated scaled copies of any linear combination of these functions, say

$$
B^{(0)}=H^{(0)}+\alpha H^{(1)}, \quad B^{(1)}=\left(\beta H^{(0)}+H^{(1)}\right) s,
$$

where we have included a scaling factor $s$ as well, to give the most general basis. One choice used in practice is $\alpha=-3, \beta=1 / 3, s=3$, which gives the $B$-spline basis:

$$
\begin{aligned}
& B^{(0)}(x)=(1-x)^{3}, \quad 0 \leqslant x \leqslant 1, \quad B^{(1)}(x)=(x-1)^{2}(5 x+1), \quad 0 \leqslant x \leqslant 1, \\
& =B^{(1)}(-x), \quad-1 \leqslant x \leqslant 0, \quad=B^{(0)}(-x), \quad-1 \leqslant x \leqslant 0 .
\end{aligned}
$$

These have the property of being positive throughout the interval of support.

In what follows we shall discuss the problem of choosing $\alpha, \beta, s$ to minimize the condition number of the Gram matrix. Because of the two basic basis functions and the fact that the support is two subintervals, the Gram matrix has the blockToeplitz form 


$$
G=h\left(\begin{array}{lll}
A & C & \\
C^{T} & A & C
\end{array}\right),
$$

where $A$ and $C$ are $2 \times 2$ blocks. (For the general Hermite case, $A$ and $C$ are $n \times n$.) The spectrum of the doubly infinite version of this is given by the set of eigenvalues of the $2 \times 2$ positive definite Hermitian matrix

$$
P(\theta)=A+C e^{i \theta}+C^{T} e^{-i \theta} .
$$

Thus

$$
\kappa(\alpha, \beta, s)=\frac{\max _{\theta} \lambda_{1}(P(\theta))}{\min _{\theta} \lambda_{2}(P(\theta))} .
$$

First, we consider $\alpha=\beta=0$, that is, using the natural Hermite basis with some scaling of the second function $H^{(1)}(x)$. The basic Gram matrix (with $s=1$ ) has, after a simple computation with (3.1),

$$
A=\frac{1}{420}\left(\begin{array}{cc}
312 & 0 \\
0 & 8
\end{array}\right), \quad C=\frac{1}{420}\left(\begin{array}{cc}
54 & -13 \\
+13 & -3
\end{array}\right) \text {. }
$$

This gives, except for a constant,

$$
P(\theta) \equiv P_{0}(\theta)=\left(\begin{array}{cc}
156+54 \cos \theta & -13 i \sin \theta \\
13 i \sin \theta & 4-3 \cos \theta
\end{array}\right) \text {. }
$$

Since we are scaling the second basis function (see (3.2)), and this function affects the second row and column of $A$ and $C$, we obtain

$$
A(s)=D A D^{T}, \quad C(s)=D C D^{T}, \quad P(s ; \theta)=D P_{0}(\theta) D^{T}
$$

where

$$
D=\left(\begin{array}{ll}
1 & 0 \\
0 & s
\end{array}\right)
$$

THEOREM 3.1. The scaled natural cubic Hermite basis (3.1) has condition $\kappa(s)$

$\geqslant 7$. This minimum is achieved for $s_{1}^{2}<s^{2}<s_{2}^{2}$, where $s_{1}^{2} \simeq 32, s_{2}^{2} \simeq 96$.

Proof. From the above, we have

$$
P(\theta ; s)=\left(\begin{array}{cc}
156+54 \cos \theta & (-13 i \sin \theta) s \\
(13 i \sin \theta) s & (4-3 \cos \theta) s^{2}
\end{array}\right) .
$$

Since $P(\theta ; s)$ is positive definite, we can denote the larger by $\lambda_{1}(\theta ; s)$, the smaller by $\lambda_{2}(\theta ; s)$, and both are always positive. Since $\lambda_{1}(\theta ; s)$ is larger than any diagonal element, and $\lambda_{2}(\theta ; s)$ is correspondingly smaller,

$$
\lambda_{2}(\theta ; s) \leqslant(4-3 \cos \theta) s^{2} \leqslant \lambda_{1}(\theta ; s) .
$$

Thus, 


$$
\kappa(s)=\frac{\max _{\theta} \lambda_{1}(\theta ; s)}{\min _{\theta} \lambda_{2}(\theta ; s)} \geqslant \frac{\max _{\theta}(4-3 \cos \theta) s^{2}}{\min _{\theta}(4-3 \cos \theta) s^{2}}=7 .
$$

To show the range where this is actually achieved is more difficult. We have explicitly

$$
\begin{aligned}
& 2 \lambda_{1}(\theta ; s)=F+\sqrt{D}, \quad 2 \lambda_{2}(\theta ; s)=F-\sqrt{D}, \\
& F=4\left(39+s^{2}\right)+3 \cos \theta\left(18-s^{2}\right), \\
& D=\left[4\left(39-s^{2}\right)+3 \cos \theta\left(18+s^{2}\right)\right]^{2}+676 s^{2} \sin ^{2} \theta .
\end{aligned}
$$

First, we compute these eigenvalues at the endpoints $\theta=0$ and $\pi$. (Since they are functions of $\cos \theta$, we need only consider the range $0 \leqslant \theta \leqslant \pi$.) We have

$$
\begin{array}{ll}
2 \lambda_{1}(0)=210+s^{2}+\left|210-s^{2}\right|, & 2 \lambda_{2}(0)=210+s^{2}-\left|210-s^{2}\right|, \\
2 \lambda_{1}(\pi)=102+7 s^{2}+\left|102-7 s^{2}\right|, & 2 \lambda_{2}(\pi)=102+7 s^{2}-\left|102-7 s^{2}\right| .
\end{array}
$$

Thus,

$$
\begin{array}{ll}
2 \lambda_{1}(0)=\max \left(420,2 s^{2}\right), & 2 \lambda_{2}(0)=\min \left(420,2 s^{2}\right), \\
2 \lambda_{1}(\pi)=\max \left(204,14 s^{2}\right), & 2 \lambda_{2}(\pi)=\min \left(204,14 s^{2}\right) .
\end{array}
$$

Hence,

$$
\begin{aligned}
\max \left(2 \lambda_{1}(0), 2 \lambda_{1}(\pi)\right) & =420\left(=2 \lambda_{1}(0)\right) & & \text { for } s^{2} \leqslant 30 \\
& =14 s^{2}\left(=2 \lambda_{1}(\pi)\right) & & \text { for } s^{2} \geqslant 30 \\
\min \left(2 \lambda_{2}(0), 2 \lambda_{2}(\pi)\right) & =2 s^{2}\left(=2 \lambda_{2}(0)\right) & & \text { for } s^{2} \leqslant 102, \\
& =204\left(=2 \lambda_{2}(\pi)\right) & & \text { for } s^{2} \geqslant 102 .
\end{aligned}
$$

So we see immediately that for $s^{2}<30$ and $s^{2}>102$,

$$
\kappa(s)=\frac{\max _{\theta} \lambda_{1}(\theta ; s)}{\min _{\theta} \lambda_{2}(\theta ; s)} \geqslant \frac{\max \left(\lambda_{1}(0), \lambda_{1}(\pi)\right)}{\min \left(\lambda_{2}(0), \lambda_{2}(\pi)\right)}>7 .
$$

However, the right-hand ratio is exactly 7 for $30 \leqslant s^{2} \leqslant 102$; and this will equal $\kappa(s)$ if the max and min are achieved at the endpoints. To examine this, rewrite $D$ as a function of $\cos \theta$ :

$$
D=a \cos ^{2} \theta+2 b \cos \theta+c,
$$

with $a=16\left(39-s^{2}\right)^{2}+26^{2} s^{2}, b=12\left(18+s^{2}\right)\left(39-s^{2}\right), c=9\left(18+s^{2}\right)^{2}-$ $26^{2} s^{2}$. So as functions of $\cos \theta$, for fixed $s$,

$$
\begin{aligned}
2 \lambda_{1}^{\prime} & =F^{\prime}+\frac{1}{2} \frac{D^{\prime}}{\sqrt{D}}, \quad 2 \lambda_{2}^{\prime}=F^{\prime}-\frac{1}{2} \frac{D^{\prime}}{\sqrt{D}} \\
2 \lambda_{1,2}^{\prime \prime} & = \pm D^{-3 / 2}\left(D D^{\prime \prime} / 2-\left(D^{\prime} / 2\right)^{2}\right) .
\end{aligned}
$$

Computing this term in parentheses, we find

$$
2 \lambda_{1,2}^{\prime \prime}(\cos \theta)= \pm D^{-3 / 2}\left(a c-b^{2}\right) \text {. }
$$

So the signs of $\lambda_{1}^{\prime \prime}, \lambda_{2}^{\prime \prime}$ are fixed for all $\theta$, and they are opposite. In other words, for each fixed $s$, one eigenvalue is a convex function of $\cos \theta$, and the other is concave. 
And a brief computation gives

$$
a c-b^{2}=-7 \cdot 26^{2} s^{2}\left(s^{4}-128 s^{2}+36 \cdot 85\right) .
$$

Thus when the quadratic in $s^{2}$ is negative (which occurs for $s_{1}^{2} \leqslant s^{2} \leqslant s_{2}^{2}, s_{1}^{2}=$ $\left.64-2 \sqrt{259} \cong 32, s_{2}^{2}=64+2 \sqrt{259} \cong 96\right), \lambda_{1}(\cos \theta)$ is convex and $\lambda_{2}(\cos \theta)$ is concave; thus, in this region the max of $\lambda_{1}$ and min of $\lambda_{2}$ must occur at the endpoints $(\theta=0$ or $\pi)$. Thus for $s_{1}^{2}<s^{2}<s_{2}^{2}$,

$$
\kappa(s)=\frac{\max \left(\lambda_{1}(0), \lambda_{1}(\pi)\right)}{\min \left(\lambda_{2}(0), \lambda_{2}(\pi)\right)}=\frac{\lambda_{1}(\pi)}{\lambda_{2}(0)}=7 \text {. Q.E.D. }
$$

Now we consider the general basis (3.2). Because of the way this is formed, the corresponding $P$-matrix

$$
P(\alpha, \beta, s ; \theta)=S P_{0}(\theta) S^{T}, \quad S=\left(\begin{array}{cc}
1 & \alpha \\
\beta s & s
\end{array}\right),
$$

and the problem now is to find

$$
\min _{\alpha, \beta, s} \kappa(P)=\min _{\alpha, \beta, s}\left[\frac{\max _{\theta} \lambda_{1}\left(S P_{0}(\theta) S^{T}\right)}{\min _{\theta} \lambda_{2}\left(S P_{0}(\theta) S^{T}\right)}\right] .
$$

TheOREM 3.2. For the general cubic Hermite basis (3.2), the condition number $\kappa(P) \geqslant 7$.

Proof. First, we decompose $S$ into $Q R$ factors ( $Q$ orthogonal, $R$ upper triangular), so that $P=Q R P_{0} R^{T} Q^{T}$; and we can reduce the problem to

$$
\min _{\alpha, \beta, s} \kappa\left(Q^{T} P Q\right)=\min _{\alpha, \beta, s} \kappa\left(R P_{0} R^{T}\right),
$$

since this matrix has the same eigenvalues as $P$. In fact, we have

$$
R=\left(\begin{array}{ll}
p & r \\
0 & q
\end{array}\right)=\frac{1}{\sqrt{1+\beta^{2} s^{2}}}\left(\begin{array}{cc}
1+\beta^{2} s^{2} & \alpha+\beta s^{2} \\
0 & s(\alpha \beta-1)
\end{array}\right) .
$$

Clearly, there is a one to one relationship between the triples $(\alpha, \beta, s)$ and $(p, q, r)$, providing we keep $p \geqslant 1$. So we can reformulate our problem (3.5) as

$$
\min _{R}\left[\frac{\max _{\theta} \lambda_{1}\left(R P_{0} R^{T}\right)}{\min _{\theta} \lambda_{2}\left(R P_{0} R^{T}\right)}\right] .
$$

Now, we decompose $P_{0}(\theta)$ into triangular factors $P_{0}(\theta)=U U^{*}$. From the definition of $P_{0}(\theta)$, this gives

$$
U=\left(\begin{array}{ll}
x & i y \\
0 & z
\end{array}\right)=\frac{1}{\sqrt{8-6 \cos \theta}}\left(\begin{array}{cc}
\sqrt{7\left(65-36 \cos \theta+\cos ^{2} \theta\right)} & -13 i \sin \theta \\
0 & 4-3 \cos \theta
\end{array}\right) .
$$

Thus, $R P_{0} R^{T}=(R U)(R U)^{*}$, with

$$
R U=\left(\begin{array}{cc}
p x & r z+i p y \\
0 & q z
\end{array}\right)
$$


Now

$$
\max _{\theta} \lambda_{1}\left(R P_{0} R^{T}\right)=\max _{\theta}\|R U\|_{2}^{2} \geqslant \max _{\theta}(q z(\theta))^{2}
$$

and

Thus,

$$
\min _{\theta} \lambda_{2}\left(R P_{0} R^{T}\right)=\min _{\theta}\left\|(R U)^{-1}\right\|_{2}^{-2} \leqslant \min _{\theta}(q z(\theta))^{2}
$$

$$
\min _{R} \kappa(P) \geqslant \frac{\max _{\theta}(z(\theta))^{2}}{\min _{\theta}(z(\theta))^{2}}=\frac{\max _{\theta}(4-3 \cos \theta)}{\min _{\theta}(4-3 \cos \theta)}=7 \text { Q.E.D. }
$$

This minimum condition of 7 is in fact attained for other choices of $\alpha, \beta$, and $s$. Suppose $\alpha$ and $\beta$ are of opposite sign, and we choose $s^{2}=s_{0}^{2}=-\alpha / \beta(>0)$. Then the matrix $R$ used in the proof of Theorem 3.2 is

$$
R=\sqrt{1-\alpha \beta}\left(\begin{array}{cc}
1 & 0 \\
0 & -s_{0}
\end{array}\right)
$$

and the corresponding condition number $\kappa$ is the same as that for the diagonally scaled matrix

$$
\left(\begin{array}{ll}
1 & 0 \\
0 & s_{0}
\end{array}\right) P_{0}(\theta)\left(\begin{array}{ll}
1 & 0 \\
0 & s_{0}
\end{array}\right)
$$

which is $P\left(\theta ; s_{0}\right)$ in (3.4). Thus from Theorem 3.1 , if $s_{1}^{2}<s_{0}^{2}=-\alpha / \beta<s_{2}^{2}$, this scaling produces a matrix with $\kappa=7$.

Now, we return to the $B$-spline basis $(\alpha=-3, \beta=1 / 3, s=3)$. It is fairly easy to check that this value of $s$ does give the minimal condition for this choice of $\alpha$ and $\beta$ (the two basic basis functions $B^{(0)}(x), B^{(1)}(x)$ are then mirror images). And since $s^{2}=-\alpha / \beta$, this condition number is the same as the diagonally scaled matrix $P(\theta ; 3)$ in (3.4). From the proof of Theorem 3.1, the eigenvalues of this are

$$
2 \lambda_{1}(\theta ; 3)=F+\sqrt{D}, \quad 2 \lambda_{2}(\theta ; 3)=F-\sqrt{D},
$$

where

$$
F=192+27 \cos \theta, \quad D=(120+81 \cos \theta)^{2}+78^{2} \sin ^{2} \theta .
$$

A brief computation shows that $\lambda_{1}(\theta ; 3)$ is maximized at $\theta=0$ and $\lambda_{2}(\theta ; 3)$ is minimized at $\theta=0$, giving $\kappa(P(\theta ; 3))=70 / 3$.

Thus the condition number of the $B$-spline basis is slightly higher than for the (properly scaled) natural basis (although both are perfectly acceptable for normal floating-point computation). However, we should emphasize that this analysis holds only for an equally spaced mesh; for an arbitrary mesh the situation may be completely different.

To conclude, we computed the condition numbers for various finite versions of natural and $B$-spline bases for $C^{1}$ cubics (by computing the condition numbers of the appropriate finite segments of the Gram matrix). The results were as follows: 
natural basis $(\alpha=\beta=0)$

$B$-spline basis $(\alpha=-3, \beta=1 / 3)$

$\begin{array}{cccccccccc}s \mid n & 10 & 20 & 30 & 40 & s \mid n & 10 & 20 & 30 & 40 \\ 1 & 165 & 195 & 203 & 206 & 3 & 18.6 & 21.9 & 22.6 & 22.9 \\ 5 & 6.96 & 7.98 & 8.19 & 8.28 & 1 & 55.2 & 64.3 & 66.5 & 67.4 \\ 6 & 5.83 & 6.64 & 6.83 & 6.90 & & & & & \\ 9 & 6.29 & 6.78 & 6.90 & 6.94 & & & & & \\ 10 & 6.69 & 6.96 & 7.02 & 7.04 & & & & & \end{array}$

As Theorem 3.1 indicates, the condition of the natural basis increases to 7.0 as $n \rightarrow \infty$ for any $s$ with $\sqrt{32} \leqslant s \leqslant \sqrt{96}$. This is also interesting from a matrix scaling point of view: as $n \rightarrow \infty$, the best diagonal scaling $D G D$ is not unique; yet for any finite $n$, since the matrix $G$ has block-tridiagonal form with the diagonal blocks themselves diagonal matrices, the best scaling of $G$ is such that the diagonal elements are equal (i.e. $s=\sqrt{39}$ ); see for example Forsythe and Straus [5] .

Department of Computer Science

The University of British Columbia

Vancouver, B. C., Canada V6T 1 W5

1. CARL de BOOR, "On calculating with B-splines," J. Approximation Theory, v. 6, 1972, pp. 50-62. MR 49 \#3381.

2. M. H. SCHULTZ, Spline Analysis, Prentice-Hall, Englewood Cliff, N. J., 1973. MR 50 \#15270.

3. G. STRANG \& G. FIX, An Analysis of the Finite Element Method, Prentice-Hall, Englewood Cliffs, N. J., 1973.

4. I. J. SCHOENBERG, "Cardinal interpolation and spline functions," J. Approximation Theory, v. 2, 1969, pp. 167-206. MR 41 \#266.

5. G. E. FORSYTHE \& L. W. STRAUS, “On best conditioned matrices," Proc. Amer. Math. Soc., v. 6, 1955, pp. 340-345. MR 16, 1054. 